\title{
On-Off Period Mechanism for Supporting Always Best Connected in IEEE 802.21 MIH Services
}

\author{
Nawawit Nounkaw, Aphirak Jansang, and Anan Phonphoem
}

\begin{abstract}
IEEE 802.21 MIH standard defines the mechanism for allowing a mobile device with more than one interface to seamlessly roam between different network technologies. All interfaces are simultaneously turned on for discovering the candidate technologies which cause unnecessary power consumption. In this paper, an On-Off Period (OOP) mechanism for supporting always best connected (ABC) has been proposed by enhancing the monitoring process without any centralized information requirement. The study shows that for the random waypoint mobility pattern and speeds between 1 - $3 \mathrm{~m} / \mathrm{s}$, the low off-durations ( 15 - 30 seconds) significantly save the energy around $54 \%$ with low loss opportunity.
\end{abstract}

Index Terms-MIH, IEEE 802.21, power consumption, On-Off Period mechanism (OOP), ABC

\section{INTRODUCTION}

Currently in a wireless coverage area, accessing to the network can be done through various available technologies such as IEEE 802.11 (WiFi), universal mobile telecommunications systems (UMTS), IEEE 802.16 (WiMAX), and long term evolution (LTE) [1]. To appropriately access those networks, the mobile device requires multiple radio interfaces. A connecting device also requires a selection criterion and handover mechanisms to support the always best connected (ABC) [1], [2].

Since 2008, the media independent handover (MIH) services have been rectified by the IEEE 802.21 standard to allow any mobile device to seamlessly roam between different network technologies [3]. However, the standard is still facing with certain issues such as high handover latency, inappropriate selection mechanism and parameters, ping-pong effects between technologies, and unnecessary power consumption by multiple radio interfaces [4].

In the IEEE 802.21 MIH standard, each interface operates in either active or standby mode. In active mode, only one interface will be used for receiving and transmitting data. In contrast with the standby mode, non-active interfaces keep monitoring theirs available receiving signal strength which can be used as candidates for handover process in case of the active technology is not currently available or less appropriate. Obviously for the limited power source of a mobile device, more standby interfaces can consume more energy.

In Section II of the paper, the background and related work on power management in IEEE 802.21 MIH services have been presented. Section III shows the proposed On-Off

Manuscript received December 25, 2012; revised February 21, 2013

The authors are with the Department of Computer Engineering, Kasetsart University, Bangkok, Thailand (e-mail: \{g521455016, aphirak.j, anan.p\}@ku.ac.th).
Period (OOP) mechanism for supporting ABC in IEEE 802.21 MIH services. The performance evaluation of on-off periods, power consumption, and loss of connectivity opportunity for the standby interfaces has been shown in Section IV. Finally, Section V concludes the paper.

\section{BACKGROUND AND RELATED WORK}

\section{A. IEEE 802.21 MIH Standard Overview}

The standard is designed for supporting mobile devices to facilitate handover between IEEE 802 networks and non IEEE 802 networks, including both wired and wireless. MIH functions are implemented between Data Link and Network layer, in both mobile device and core network. Three main services, as shown in Fig. 1, have been defined for supporting handovers between heterogeneous access links: media independent event service (MIES), media independent command service (MICS) and media independent information service (MIIS).

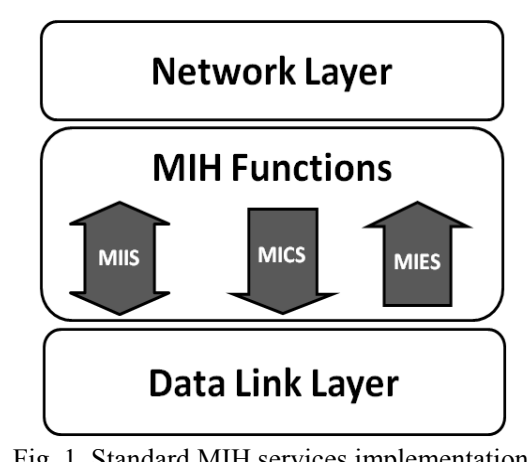

MIES monitors the Data Link status, such as Link Going Down and Link Up/Down, and then reports back to Network Layer. The MIH device sends command such as handover initiation to Data Link Layer via the MICS, while MIIS provides the information about all other networks at the current position.

\section{B. Related Work on Power Management}

Power management researches in MIH service can be classified into two groups: power awareness during handover decision and power consumption reduction of standby interfaces.

For power awareness during handover decision, many researchers proposed mechanisms to choose a new active interface when the parameters such as cost or power level hit certain criterion [5]-[7]. However, the standby interfaces still normally consume the power. The interface selection algorithms and call admission control (CAC) has been proposed by considering power source level and distance 
between a mobile device and base station (BS) in overlay network [6]. The fuzzy set representation (FSR) TOPSIS method [7] are subjected to support handover decision of real-time and non-real-time applications by avoiding possible inconsistency of ranking results of candidate technologies.

For power consumption reduction, some researchers focus on reducing power use of standby interfaces by turning them off for some intervals, while the interface operated in the active mode is an always-on interface [8]-[10]. During the mobility situation, the number of handovers and the number of turn-on times for standby interfaces has been studied [8]. However, when the movement speed was over a suitable connection threshold, for the particular technology, the interface should be turned off [9]. Some interfaces should also be tuned off when the devices power level becomes critically low.

Furthermore, the work in the power reduction category can be divided into the centralized and decentralized manner. In the centralized manner, a central server provides some useful information, such as available services in the coverage area, for assisting a mobile device to turn off unnecessary standby interfaces.

A paging mechanism [11] has been proposed that a mobile device only turns on the active interface (other interfaces have been turned off) and keeps updating its own location to the server. Once the device enters a certain covered zone, the mobile device will be paged to turn on the particular interface. By receiving the location information of $\mathrm{WiFi}$ access point from a central server, the on-off patterns of the standby interfaces are proposed, However, some work do not require any information from a centralized server [8], [9].

In this paper, the effects of on-off periods, power consumption, and loss of connectivity opportunity for the standby interfaces during the MIH service without a centralized server have been analyzed.

\section{On-OfF PERIOd Mechanism For SupPORTING ABC}

The main goal for our study is to decrease the power consumption in the MIH service of all standby interfaces by assigning the on-off pattern for the particular interface with ABC awareness. To be able to define the suitable on-off period called OOP (shown in Fig. 2) to each interface, the 802.21MIH standard is slightly modified as shown in Fig. 3.

After a standby interface turns off for $n$ seconds, it will turn on for one second to monitor the available network. If the link to the network has been detected, it will enter the Get Information Phase by using MIIS to retrieve the related information. Then, the Decision Phase will use the retrieved information for making handover decision and enters the Handover Phase as in the regular process. However, if no link available, the interface will remain in the OOP state by switching itself to off state for another $n$ seconds off-duration.

When a mobile device is leaving out of the current coverage area, triggered by the Link Going Down event by the MIES, it will change to the regular period state by turning on all standby interfaces for preparation to the nearby incoming handover. Once the handover process is over, the mobile device enters the OOP state. All non-active interfaces become standby interfaces. The state transition can be shown by Fig. 4 .

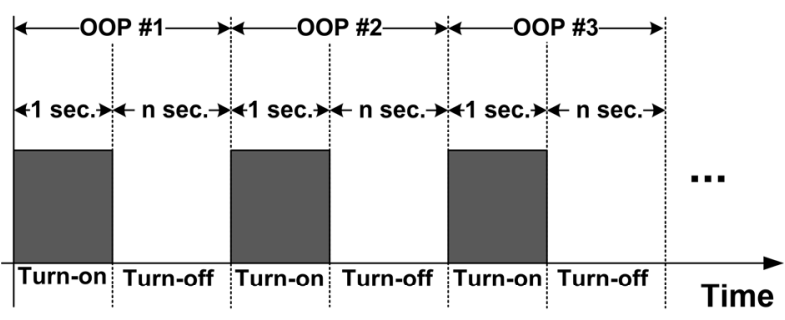

Fig. 2. On and off-durations in the OOP mechanism

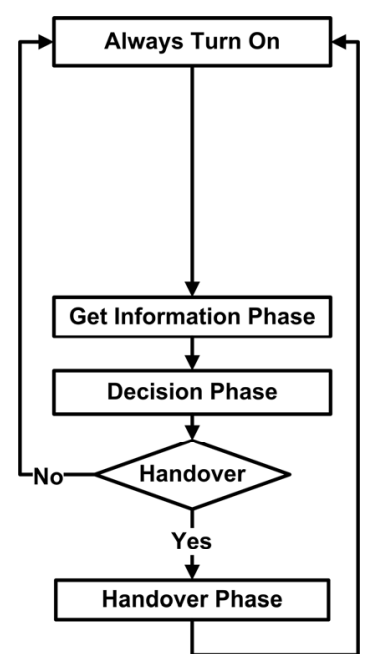

(a) Regular mechanism

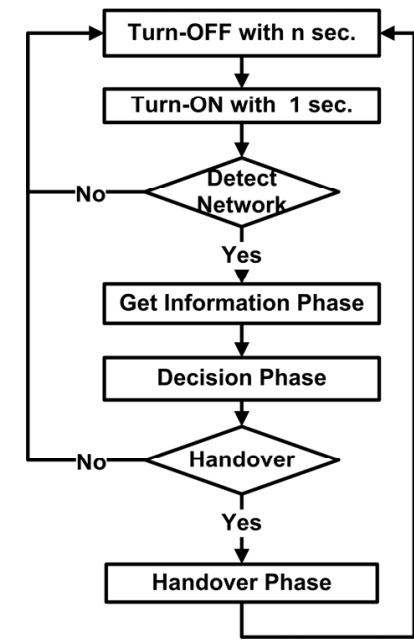

(b) OOP mechanism Fig. 3. The regular and the proposed OOP mechanism comparison

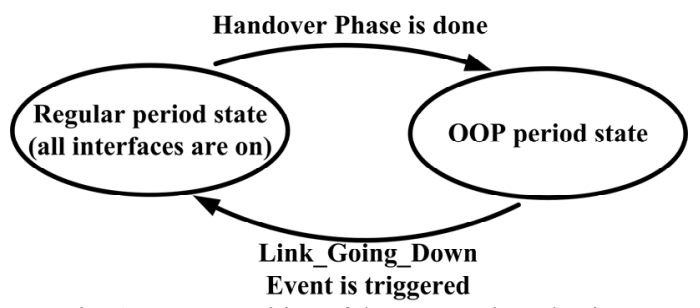

Fig. 4. State Transition of the proposed mechanism

\section{Performance Evaluation}

With the proposed OOP scheme, the detection of a new available wireless technology at the cell edge might be delayed when compared with the regular scheme that all interfaces are always-on.

\section{A. Loss Opportunity}

To evaluate the $\mathrm{ABC}$, the loss opportunity has been studied. Fig. 5 shows the loss opportunity definition. At position (I), a mobile device moves from the UMTS to the WiMAX coverage area. In case of the regular scheme, a new signal of the WiMAX will be immediately detected, while in OOP the detection might be delayed, position (II), for an $M_{x}$ seconds due to the current status of the standby interface (WiMAX) is off. The mobile device remains connected with the WiMAX until reaching the edge of the WiFi cell at position (III). The detection of the WiFi signal might also be delayed for $F_{x}$ seconds. Therefore, the loss opportunity $(L)$ of the WiMAX and WiFi can be calculated as (1) and (2), respectively. 


$$
\begin{aligned}
L_{W i M A X} & =\frac{M_{x}}{M_{T 1}+M_{T 2}} \\
L_{W i F i} & =\frac{F_{x}}{F_{T}}
\end{aligned}
$$

\section{B. Experimental Setup}

The behavior of the proposed mechanism in the MIH services has been studied by implementing on the network simulator (NS) version 2.29 [12] with the mobility patch written by NIST [13].

The test scenario has been setup as shown in Fig. 6. The test area is defined as $500 \mathrm{~m} \times 500 \mathrm{~m}$. There are three available networks (WiFi, WiMAX, and UMTS) in test area, connected to a router. By implementing a random way point mobility pattern, a mobile device starts with a random position and moves around through the coverage area of each wireless technology, while communicating data with the sink node located outside the area.

Table I shows simulation parameters. The power consumptions [8] used in the calculation are shown in Table II.

The experiment has been tested with 9 scenarios, randomly selected starting positions in the test area. Each scenario is evaluated for the OOP with 5, 10, 15, 30, and 60 seconds off-durations. Fig. 7 shows an example of experiments with a starting location and movement trace. Each starting point will be repeatedly experimented with three constant moving speeds: 1,2 and $3 \mathrm{~m} / \mathrm{s}$.

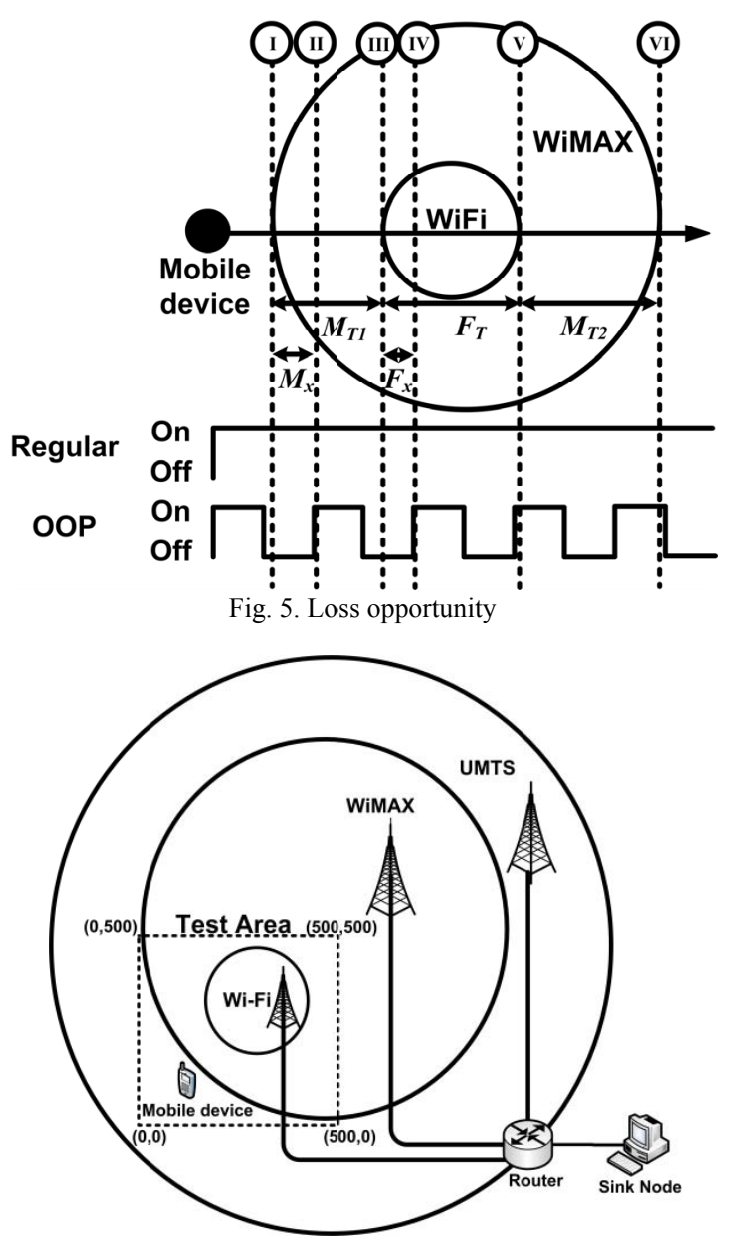

Fig. 6. Network topology for simulation

\section{Results and Discussion}

Fig. 8 shows active interfaces during the corresponding movement of Fig. 7. In Fig. 8(a), the active interface utilization of the regular scheme is shown, while Fig. 8(b)-(f) represent the active interface utilization of OOP scheme with certain loss opportunity of WiMAX and WiFi.

Fig. 9 demonstrates the loss opportunity of the standbyWiFi interface with the various on-off periods. For example, in 15 second off-duration shown in Fig. 9(d), at time position (I) the WiFi detection delay is 14.0002 seconds, while at the position $(\mathrm{V})$ the $\mathrm{WiFi}$ detection delay becomes 3.0002 seconds.

TABLE I: SIMULATION PARAMETERS

\begin{tabular}{ll}
\hline \multicolumn{2}{c}{ TABLE I: SIMULATION PARAMETERS } \\
\hline Parameters & Description \\
\hline Timulation time & 3,600 seconds \\
Mobile device interfaces & $500 \mathrm{~m} \times 500 \mathrm{~m}$ \\
Number of mobile device(s) & UMTS, WiMAX and WiFi \\
Number of UMTS base station(s) & 1 mobile device \\
Number of WiMAX base & 1 base station \\
station(s) & 1 AP \\
Number of WiFi access point(s) & CBR(256 kbps), 1000-byte \\
Application/Traffic & pkt. size \\
\hline \hline
\end{tabular}

\begin{tabular}{|c|c|c|c|c|c|c|}
\hline \multirow{2}{*}{$\begin{array}{l}\text { Netwo } \\
\text { rk }\end{array}$} & \multirow{2}{*}{$\begin{array}{l}\text { Range } \\
(\mathrm{m})\end{array}$} & \multicolumn{3}{|c|}{$\begin{array}{l}\text { Power consumption }(\mathrm{W}) \\
{[8]}\end{array}$} & \multicolumn{2}{|c|}{ Transition } \\
\hline & & $\mathrm{Tx}$ & $\mathrm{Rx}$ & Idle & $\begin{array}{l}\text { Power } \\
(\mathrm{W})\end{array}$ & $\begin{array}{l}\text { Time } \\
(\mathrm{sec})\end{array}$ \\
\hline UMTS & All area & 2.805 & 0.495 & 0.082 & 0.002 & 0.05 \\
\hline $\begin{array}{l}\text { WiMA } \\
\quad \mathrm{X}\end{array}$ & 500 & 1.32 & 0.66 & 0.2 & 0.002 & 0.05 \\
\hline Wi-Fi & 100 & 2.25 & 1.35 & 0.75 & 0.002 & 0.05 \\
\hline
\end{tabular}

\section{TABLEI II: CHARACTER PARAMETERS}

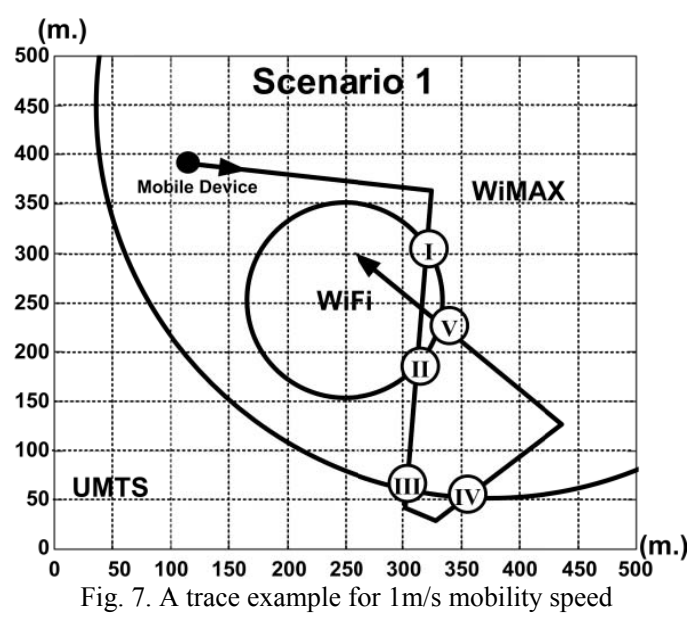

In Fig. 10(a), for the moving speed of $1 \mathrm{~m} / \mathrm{s}$, the total energy use of the mobile device for each OOP normalized with the energy use of the regular scheme, called Energy Ratio, have been displayed. The decreasing rate (the slope between each off-duration) of energy ratio is significantly high in low off-durations $(5,10$, and 15 seconds with the energy saving of $46.77 \%, 51.18 \%$, and $53.53 \%$, respectively). However, for higher off-durations (30 and 60 seconds with the energy saving of $55.63 \%$ and $57.14 \%$, respectively) the decreasing rate of energy ratio becomes slightly low.

Fig. 10(b) shows that both WiFi and WiMAX loss 
opportunity rates of low-durations are quite small. And its rate increases when the off-durations getting higher. The increasing of WiMAX loss opportunity rate is slower than the WiFi loss opportunity rate due to the mobile device stays longer in the WiMAX coverage area. In the current scenario, from (1) and (2), the $M_{x}$ and $F_{x}$ are quite the same, while $M_{T 1}+M_{T 2}$ is much greater than the $F_{T}$ durations.

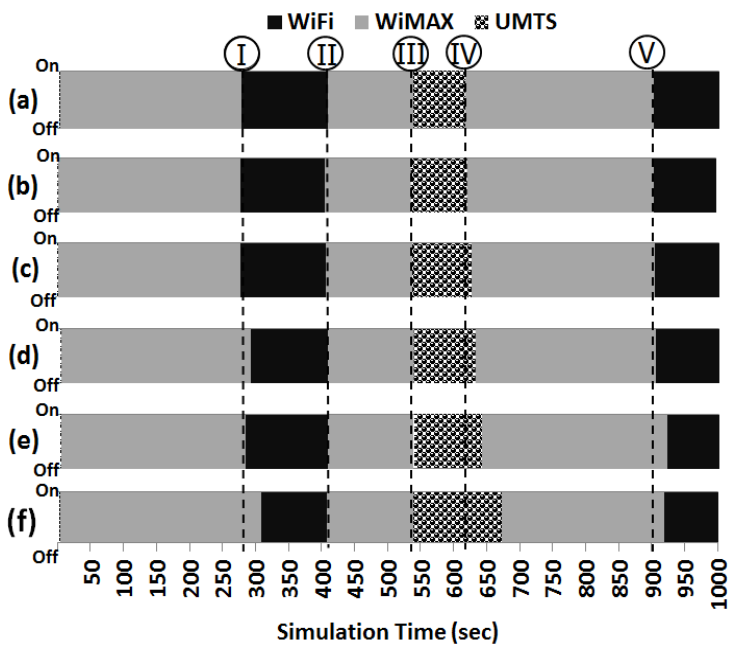

Fig. 8. The active interface utilization (from Fig. 7 scenario) for various off-durations

(a) $0 \mathrm{sec}$, (b) $5 \mathrm{sec}$, (c) $10 \mathrm{sec}$, (d) $15 \mathrm{sec}$, (e) $30 \mathrm{sec}$, (f) $60 \mathrm{sec}$

$$
\text { (I) (II) (III) (IV) }
$$

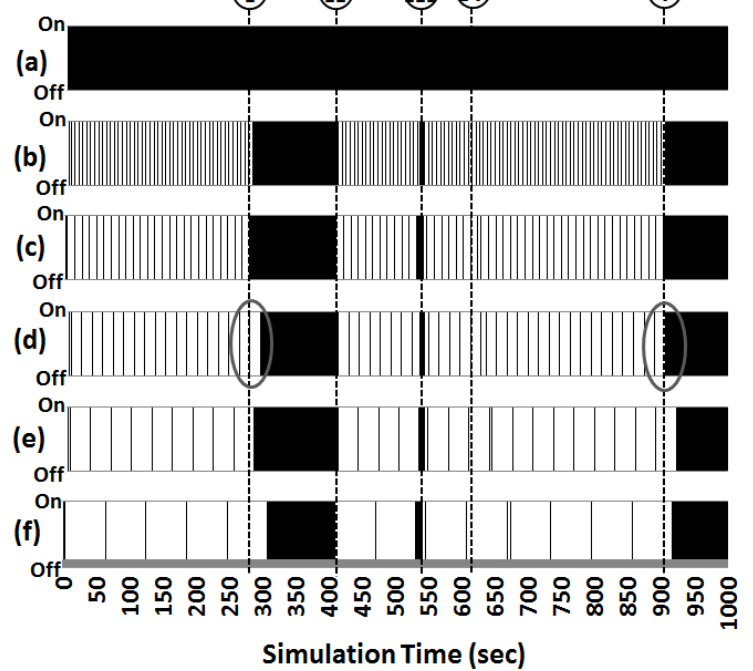

Fig. 9. The status of WiFi interfaces (from Fig. 7 scenario) for various off-durations

(a) $0 \mathrm{sec}$, (b) $5 \mathrm{sec}$, (c) $10 \mathrm{sec}$, (d) $15 \mathrm{sec}$, (e) $30 \mathrm{sec}$, (f) $60 \mathrm{sec}$

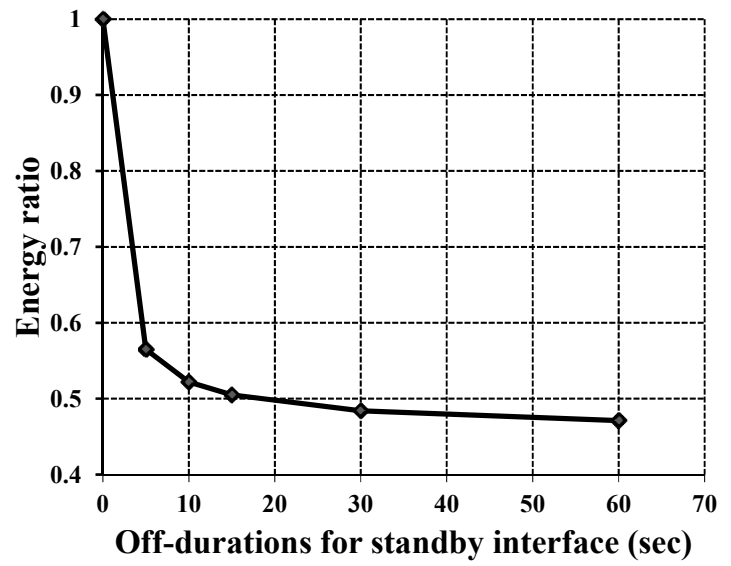

(a)

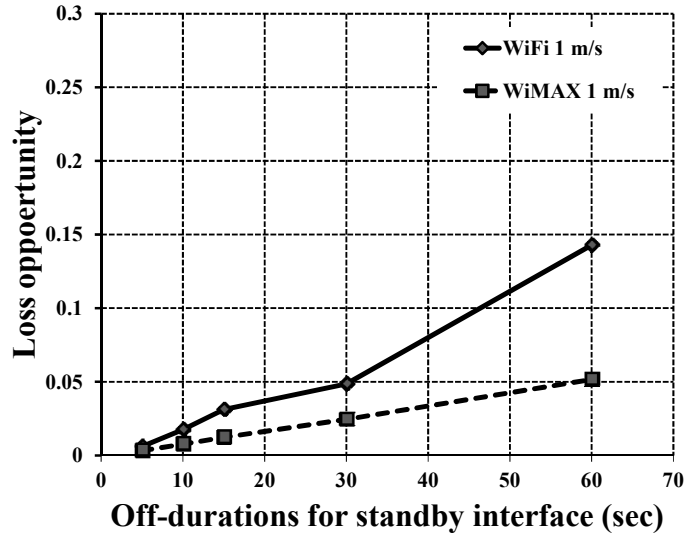

(b)

Fig. 10. Experimental results for $1 \mathrm{~m} / \mathrm{s}$ mobility speed (a) energy ratio and (b) loss opportunity

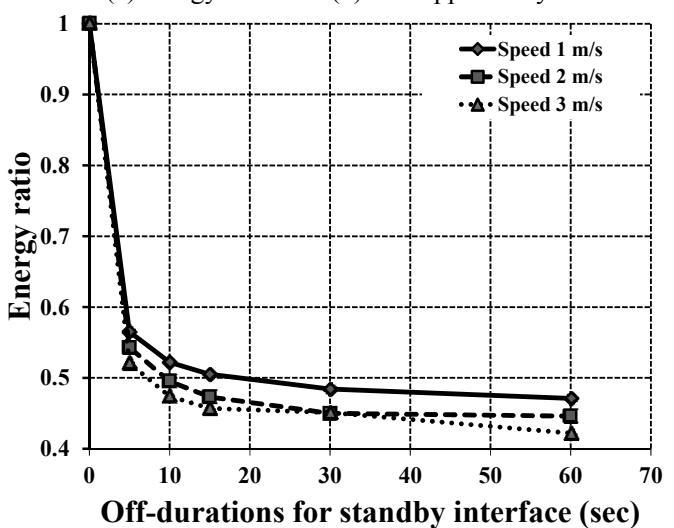

(a)

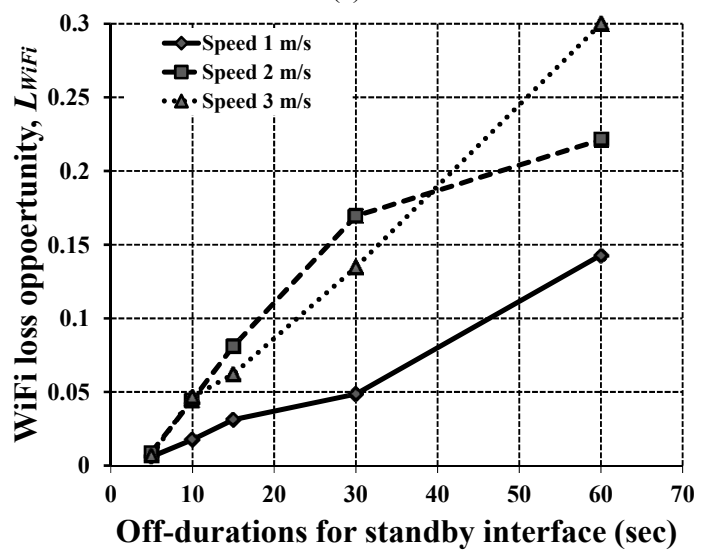

(b)

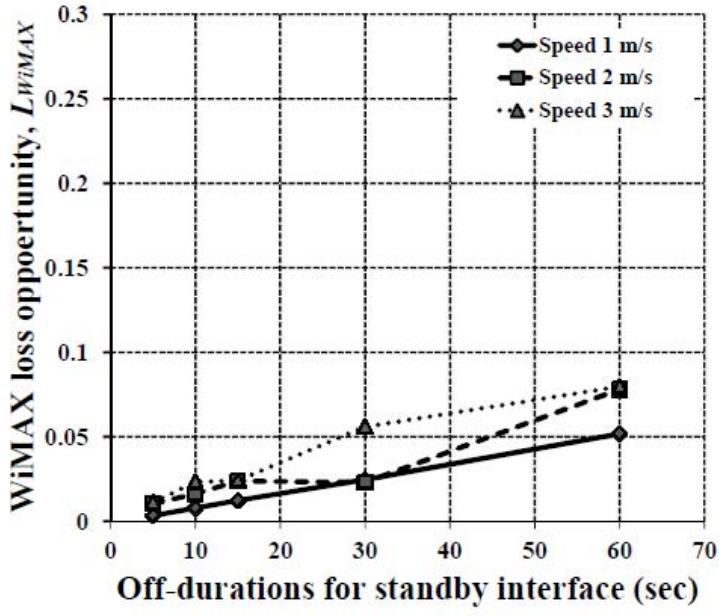

(c)

Fig. 11. Experimental results for various mobility speeds (a) energy ratio (b) WiFi loss opportunity (c) WiMAX loss opportunity 
The results show that the decreasing rate of energy ratio for each speed $(1,2$, and $3 \mathrm{~m} / \mathrm{s})$ holds the same trend, as show in Fig. 11(a). Once the speed of mobile device increases, the WiFi loss opportunity rate becomes higher, while the increasing of WIMAX loss opportunity rate does not significantly change as shown in Fig. 11(b) and 11(c) respectively.

Therefore, the off-duration has the significantly influences on the power consumption only for low value of off-duration with low loss opportunity. For higher off-durations, the power is not significantly saved while it may cause higher loss opportunity. From the experiments, for gaining the best performance, the suitable off-durations should be in the range of 15 to 30 seconds which can save the energy around 54\% compared with the regular scheme.

\section{CONCLUSION}

From the proposed On-Off Period (OOP) mechanism to support always best connected (ABC) properties, the mobile device can save more energy by turning off the standby interfaces for a period of time while the chance to detect the suitable available wireless signal candidates for handover process is still an acceptable ABC with low loss opportunity. The movement speed of mobile device has higher effects on the small wireless coverage area (such as WiFi) than the higher coverage area (such as WiMAX). More mobility patterns, speeds, and the size of the coverage area for each technology are needed for investigation in the future.

\section{REFERENCES}

[1] A. B. Pontes, D. D. P. Silva, J. Jailton, O. Rodrigues, and K. L. Dias, "Handover management in integrated WLAN and mobile WiMAX networks," Wireless Communications, IEEE, vol. 15, no. 5, pp. 86-95, October 2008

[2] E. Gustafsson and A. Jonsson, "Always best connected," Wireless Communications, IEEE, vol. 10, no. 1, pp. 49-55, Feb. 2003.

[3] IEEE Standard for Local and Metropolitan Area Networks- Part 21: Media Independent Handover, IEEE Std. 802.21-2008.

[4] C. L. Lin, C. H. Ho, and J. Y. Pan, "Hand-off evolution with multiple interfaces," IT Professional, vol. 10, no. 5, pp. 22-28, September-October 2008.

[5] J. Chuchuay and A. Phonphoem, "A decision model for choosing suitable wireless technologies," in Proc. The 11th National Computer Science and Engineering Conference (NCSEC 2007), 2007, pp. 363-371.

[6] M. Chowdhury, Y. M. Jang, C. S. Ji, S. Choi, H. Jeon, J. Jee, and C. Park, "Interface selection for power management in UMTS/WLAN overlaying network," in Proc. Advanced Communication Technology, 2009. ICACT 2009. 11th International Conference on, vol. 01, 2009, pp. $795-799$.
[7] I. Chamodrakas and D. Martakos, "A utility-based fuzzy TOPSIS method for energy efficient network selection in heterogeneous wireless networks," Applied Soft Computing, vol. 11, no. 4, pp. 3734 3743, June 2011.

[8] C. Moon, S. Yang, and I. Yeom, "Performance analysis of decentralized RAN (radio access network) discovery schemes for IEEE 802.21," in Proc. Vehicular Technology Conference, 2007. VTC-2007 Fall. 2007 IEEE 66 ${ }^{\text {th }}, 2007$, pp. 41-45.

[9] Q.-T. N.-Vuong, N. Agoulmine, and Y. G.-Doudane, "A user centric and context-aware solution to interface management and access network selection in heterogeneous wireless environments," Computer Networks, vol. 52, no. 18, pp. 3358-3372, December 2008.

[10] W. S. Lim, D. W. Kim, Y. J. Suh, and J. J. Won, "Efficient WLAN discovery schemes based on IEEE 802.21 MIH services in heterogeneous wireless networks," in Proc. Global Telecommunications Conference, 2008. IEEE GLOBECOM 2008. IEEE., 2008, pp. 1-5.

[11] S. Seo and J. Song, "An energy-efficient interface selection for multimode terminals by utilizing out-of-band paging channels," Telecommunication Systems, vol. 42, pp. 151-161, October 2009

[12] The network simulator - ns-2. [Online]. Available: http://www.isi.edu/nsnam/ns/

[13] Seamless and secure mobility project. [Online]. Available: http://www.nist.gov/itl/antd/emntg/ssm seamlessandsecure.cfm

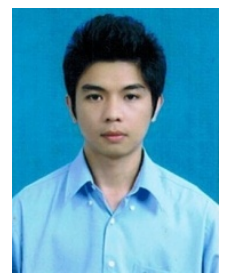

Nawawit Nounkaw received the B.Eng., degree in Computer Engineering from Prince of Songkla University, Thailand, in 2002. He is currently a Master student at the Department of Computer Engineering, Kasetsart University, Thailand.

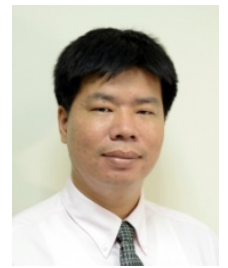

Aphirak Jansang received the B.Eng., M.Eng. and D.Eng. degrees in Computer Engineering from Kasetsart University, Bangkok, Thailand, in 2000, 2003 and 2012, respectively. He is currently a faculty member at the Department of Computer Engineering, Kasetsart University, Thailand. He is also a team leader of Intelligent Wireless Network Group (IWING). His research interests include wireless networks, resource management and QoS.

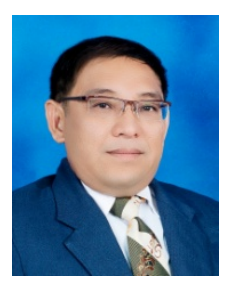

Anan Phonphoem received his Ph.D. in Electrical and Computer Engineering (2000) from University of Massachusetts Amherst, M.S. in Computer Engineering (1996) from University of Southern California, and B.Eng. in Electrical Engineering (1990) from Prince of Songkla University, Thailand. $\mathrm{He}$ is currently an Associate Professor and the director of the Intelligent Wireless Network Group (IWING) at Computer Engineering Department, Kasetsart University, Thailand. His research interests include wireless networks, ad hoc networks, performance evaluation, and protocol design and analysis. 